\title{
COMPTABILISATION DES IMMATERIELS ET MODES DE FINANCEMENT : UNE APPROCHE HISTORIQUE DE LA GESTION DU RESULTAT
}

Résumé : Cet article étudie les pratiques de gestion du résultat à partir de l'ouverture des marchés de capitaux à la fin du $19^{\text {ème }}$ siècle. A cette époque, les modes de financement des connaissent des évolutions majeures dans un contexte de réglementation comptable quasi inexistante. Une étude de cas historique de la société L'Air Liquide, réalisée de sa création à 1939, met en évidence le fait que les dirigeants utilisent la comptabilisation des dépenses incorporelles comme un instrument de gestion du résultat selon l'évolution de la structure de financement.

Mots clés : histoire, gestion du résultat, modes de financement, dépenses immatérielles, L’Air Liquide.
Abstract: This article aims at highlighting the earnings management practices from the opening of capital markets in the late 19th century. The choice of this period is due to the absence of accounting standards. On the other hand, firms benefited from a potential access to different external sources of financing. A historical case study of L'Air Liquide (1902-1939) highlights the intangible expenses as a tool for earnings management according to changes in the financing structure.

Key words: history, earnings management, intangible expenses, L'Air Liquide. 
«Manipulation comptable », « gestion du résultat», « habillage des comptes », « nettoyage des comptes », ces expressions font trembler les actionnaires, les politiques et même les commissaires aux comptes ; elles sont entrées dans le langage journalistique, et même dans le langage commun, au moment des scandales financiers du début des années 2000. L'introduction en bourse controversée de Facebook en mai 2012 a fait resurgir les démons des affaires Enron ou Worldcom et l'information comptable est apparue, une nouvelle fois, comme un outil de duperie. Loin d'être un phénomène nouveau, la gestion des données comptables et, plus précisément, la gestion du résultat, est une problématique qui a traversé les âges et qui mérite d'être étudiée en profondeur. L'ouverture des marchés des capitaux semble être un moment crucial pour en comprendre les ressorts; c'est, en effet, à ce moment que les conflits d'intérêts entre apporteurs de capitaux et dirigeants s'exacerbent et expliquent en grande partie les pratiques de gestion du résultat (Chalayer 1995 ; Mard et Marsat 2012).

C'est au cours de la dernière décennie du $19^{\text {ème }}$ siècle que le marché financier français s'ouvre aux sociétés industrielles. Nombreuses sont celles qui vont profiter de cette opportunité pour lever les fonds nécessaires au développement de leur activité. Ce recours est notamment motivé par les nouveaux besoins en termes d'équipements, induits par les nombreuses innovations techniques qui caractérisent cette période. Jusqu'alors, le secret des affaires et la crainte d'intrusion dans la gestion de leurs entreprises conduisaient les industriels à privilégier l'autofinancement. Seuls les établissements bancaires, à travers les avances de trésorerie et les escomptes accordés, apportaient des sources externes de financement à court terme.

Ce changement de mode de financement marque une rupture pour les entrepreneurs dans leur manière de concevoir l'objet de la comptabilité. D'un rôle de preuve et de garantie à l'égard des créanciers, la comptabilité doit désormais faire le lien et être un langage commun entre l'entreprise et ses parties prenantes. Dans le cadre d'une responsabilité illimitée qui prévalait jusqu'alors et qui signalait l'engagement et la prise de risque des entrepreneurs (de la Bruslerie 2014), la comptabilité pouvait demeurer une affaire privée; les créanciers disposaient de garanties régulières en raison des perspectives de liquidation qui animaient les pratiques comptables du $19^{\mathrm{e}}$ siècle. L'introduction du principe de responsabilité limitée ${ }^{1}$ qui constitue le socle juridique à partir duquel le capitalisme peut se diffuser, modifie la donne. La comptabilité comporte désormais une dimension sociale. Elle a une fonction d'information à l'égard des différentes parties prenantes de l'entreprise dont les attentes sont diverses et souvent divergentes. La figure de l'actionnaire prend progressivement l'ascendant sur les autres figures en raison de son lien direct et permanent avec l'entreprise et de sa contribution croissante au financement.

Dans ce contexte, la problématique de la détermination du résultat distribuable prend toute son importance. En l'absence de réglementation comptable, les industriels vont utiliser la comptabilité pour délimiter les contours souhaités du résultat distribuable. De tels agissements correspondent à ce qui est aujourd'hui qualifié de « gestion du résultat ».

\footnotetext{
${ }^{1}$ En France, le statut de la société anonyme est créé en 1867.
} 
Les travaux sur la gestion des résultats se sont multipliés ces dernières années et ont permis d'identifier les formes qu'elle peut prendre, les objectifs, les motivations des acteurs et les techniques, en particulier les accruals (Marmousez 2009 ; Stolowy et Breton 2003). Les plus récents s'attachent également à expliquer les irrégularités dans la distribution des résultats publiés (Vidal 2010) ou étudient l'influence de la structure et de la nature de l'actionnariat sur la gestion des résultats (Mard et Marsat 2012). Bien que riches d'enseignements, ces études, de par la méthodologie quantitative qu'elles mobilisent (régressions multiples, mesures non paramétriques, analyses statistiques multi-factorielles, etc.), ne permettent pas d'appréhender la complexité des phénomènes. Stolowy et Breton (2003) regrettent eux-mêmes que les situations des entreprises ne soient pas assez détaillées pour distinguer ce qui relève de la gestion des résultats ou pas. Etant décontextualisés, les points de références déterminés par les chercheurs peuvent être artificiels; ce qui peut être une pratique opportuniste dans une entreprise peut éventuellement, dans une autre, relever d'un comportement acceptable.

L'objet de cet article est justement de proposer une recherche contextualisée et longitudinale sur la gestion du résultat. Il s'inscrit dans la continuité des travaux de Lemarchand et Praquin (2005) et vient les compléter. Il s'agit de poursuivre l'histoire de la gestion du résultat à la fin du $19^{\text {ème }}$ siècle et au début du $20^{\text {ème }}$ siècle, au moment de l'ouverture des marchés de capitaux, en nous plaçant du côté des entreprises plutôt que de celui du droit. En outre, l'angle d'étude retenu ici est celui des techniques utilisées, en particulier celles concernant la comptabilisation des dépenses incorporelles, en lien avec l'évolution des modes de financement de l'époque.

Le cas retenu est celui d'Air Liquide de 1902 à 1939. La méthodologie retenue est pleinement historique puisqu'elle repose sur l'exploitation de sources primaires, à savoir les archives de l'entreprise étudiée.

L'article se déroule de la façon suivante. Dans une première partie, une revue de la littérature nous permettra d'une part, de présenter les modalités de la gestion du résultat comptable et, d'autre part, d'établir un lien dans une perspective historique entre la gestion du résultat et les modes de financement. Dans une seconde partie, le récit chronologique des modes de financement et des pratiques comptables de L'Air Liquide de sa création à 1939 apporte des éléments empiriques enrichissant les théories présentées précédemment. Enfin, nous tirerons de ce récit deux principaux résultats, la gestion du résultat peut recouvrir différents objectifs. De la création de cette entreprise à sa cotation en Bourse, les modalités de gestion du résultat servent un objectif entrepreneurial : la réussite d'un projet professionnel. De 1913 à 1939, les pratiques comptables viennent appuyer un intérêt économique.

\section{GESTION DU RESULTAT, COMPTABILITE ET MODES DE FINANCEMENT}

Après avoir défini les enjeux de la gestion du résultat, en lien avec les techniques comptables et les modes de financement, nous proposons une contextualisation historique de la problématique. 


\subsection{Gestion du résultat et relations d'agence}

La gestion du résultat est une des modalités de la gestion des données comptables qui recouvre plusieurs dimensions dont le nettoyage ou l'habillage des comptes, ou le lissage du résultat. Dans tous les cas, selon Stolowy et Breton (2003) «les différentes formes de GDC [Gestion de données comptables] effectuées auront tendance à augmenter ou déplacer les possibilités de transfert de richesses », répondant notamment à des objectifs divers tels que la minimisation des coûts de financement ou la maximisation de la richesse des dirigeants.

A l'instar de Degeorge et al. (1999), nous définissons la gestion du résultat comme "l'utilisation de la discrétion managériale pour influencer le résultat diffusé auprès des parties prenantes ». Il ne s'agit pas forcément, pour le dirigeant, de s'approprier la richesse par cette manœuvre et donc de se comporter de manière opportuniste, mais de profiter de l'asymétrie d'information en sa faveur pour mettre en œuvre une stratégie.

La théorie de l'agence (ou théorie politico-contractuelle) constitue le cadre théorique fréquemment mobilisé (Holthausen et al. 1995 ; Moses 1987) pour expliquer les conflits d'intérêt entre actionnaires et dirigeants, d'une part, ou entre catégories d'apporteurs de ressources, d'autre part. Selon cette approche, l'information publiée, dont le résultat comptable, intervient dans la gestion des relations entre ces différentes parties dont les intérêts sont divergents. Appliquée à la comptabilité, cette théorie permet d'expliquer les comportements des dirigeants dans la reddition des comptes et leur diffusion.

En effet, les dirigeants peuvent mettre à profit une certaine latitude dans l'application des normes, lorsque celles-ci leur laissent la possibilité de faire des choix, quant aux méthodes d'évaluation ou de comptabilisation notamment.

La littérature retient un certain nombre d'instruments de gestion du résultat (Marmousez 2009), parmi lesquels les changements de méthodes comptables, les gains ou pertes exceptionnels, les ventes ou cessions d'actifs et les accruals. Désignant l'ensemble des ajustements comptables, les accruals ${ }^{2}$ sont les instruments les plus utilisés dans la gestion du résultat, étant ce qui permet de passer d'une comptabilité de trésorerie à une comptabilité d'engagement. Ils regroupent essentiellement les dotations aux amortissements et les dotations / reprises sur provisions qui donnent lieu à la constitution de réserves.

Le choix de méthodes de valorisation des stocks est également un instrument de gestion du résultat. Ainsi, Buckmaster (1992) note que l'option pour la méthode FIFO notamment en période d'inflation permet d'afficher des niveaux de résultat supérieur. Il ajoute cependant que si les actionnaires sont en droit d'attendre des versements de dividendes supérieurs, la

\footnotetext{
${ }^{2}$ Le terme accruals est parfois appelé « variables comptables de régularisation » (Stolowy et Breton 2003). Nous avons toutefois préféré conserver le terme anglais. Il s'agit des charges et produits calculés (dotations aux amortissements et provisions, reprises sur provisions), ainsi que des éléments de la variation du besoin en fonds de roulement. Une partie de ces accruals est discrétionnaire, les normes comptables laissant des espaces de liberté sous forme de jeu d'options (par exemple pour l'évaluation des stocks) ou d'évaluations dans la comptabilité (par exemple pour la durée d'amortissement). Ces espaces de liberté donnent des marges de manœuvre aux dirigeants pour effectuer une gestion du résultat.
} 
firme doit également financer des stocks plus coûteux et par conséquent limiter les sorties de trésorerie.

Un autre axe de recherche consiste à étudier plusieurs configurations de relations d'agence pour expliquer la gestion du résultat : la relation actionnaires familiaux - autres actionnaires, la relation dirigeant-actionnaires et la relation actionnaires de contrôle-actionnaires minoritaires (Mard et Marsat 2012). La question du sens de la relation a été posée, certains auteurs ayant pu suggérer que l'entité était incitée à pratiquer la gestion du résultat afin d'accéder à des ressources nouvelles ou moins onéreuses. D'autres avancent que ce sont les apporteurs de ressources qui, en raison de leurs propres intérêts, contraignent l'entité à pratiquer la gestion du résultat (Jeanjean 2001).

Les recherches ont envisagé diverses configurations en matière de concentration du capital ou de nature des actionnaires. Ainsi en est-il de Ali et al. (2007), Faccio et Lang (2002) ou Wang (2006) qui étudient les pratiques de gestion du résultat dans les firmes familiales. Ali et al. (2007) soulignent que les problèmes d'agence sont moindres dans de telles configurations. En effet, dans les cas où elles n'exercent elles-mêmes les activités de direction, les familles peuvent opérer un contrôle direct de l'action du dirigeant dont la latitude en matière de gestion opportuniste des données comptables est, dès lors, réduite. Ainsi les firmes familiales produiraient des résultats comptables de meilleure qualité (moins d'accruals discrétionnaires, meilleure possibilité de prédiction de cash flow sur la base des résultats). Toutefois, les chercheurs soulignent que, pour préserver le caractère familial de l'actionnariat, ces firmes sont moins enclines à diffuser des informations discrétionnaires portant notamment sur les questions de gouvernance. Wang (2006), quant à lui, distingue deux effets contradictoires sur la qualité des résultats de la présence d'un actionnariat familial : l'effet d'enracinement et l'effet d'alignement. Selon le premier, la firme à actionnariat familial pratique une gestion opportuniste des résultats visant à une appropriation de la richesse par les actionnaires familiaux, qui profitent de l'asymétrie de l'information liée à leur position d'actionnaire et manager, au détriment des autres actionnaires. D'un autre côté, l'effet d'alignement pose que l'actionnariat familial est la garantie d'une publication sincère, de résultats de meilleure qualité, des comportements opportunistes étant susceptibles d'entacher la réputation familiale ainsi que la richesse et la performance à long terme de ces firmes patrimoniales.

D'autres auteurs distinguent les firmes managériales caractérisées par un actionnariat diffus et les firmes de type actionnarial (Beattie et al. 1994 ; Smith 1976). Les études offrent des résultats divergents et conduisent à s'interroger sur l'existence d'un effet de seuil (Mard et Marsat 2012). Ainsi, les pratiques de gestion de résultat des firmes de type actionnarial tendraient à s'aligner sur celles des firmes familiales et les effets d'enracinement et d'alignement tels que décrits par Wang (2006), opéreraient également, respectivement au-delà ou en deçà, d'un certain pourcentage de détention de capital par les actionnaires majoritaires.

Ces études sont riches d'enseignements, bien que leurs résultats soient parfois divergents. Les techniques comptables de gestion du résultat tout comme l'influence des configurations de financement (en particulier les types d'actionnariat) sur la gestion des résultats sont très étayées dans la littérature. Toutefois, le lien entre ces techniques et les modes de financement mérite encore d'être approfondi. Nous souhaitons faire progresser la connaissance sur le sujet, non pas en tentant d'expliquer des liens entre des variables, mais plutôt en proposant 
d'analyser ce lien en étudiant la complexité des enjeux. Le recours à l'histoire paraît pertinent ici pour appréhender la gestion du résultat dans une dynamique nécessitant d'être contextualisée et étudiée sur une longue période.

\subsection{Mode de financement, comptabilisation des actifs et gestion du résultat : une approche historique}

Pour explorer la problématique du financement, de la comptabilité et de la gestion du résultat dans une perspective historique, nous proposons d'abord d'exposer la contradiction quant à la détermination du résultat, inhérente au double mode de financement des entreprises, à partir du $19^{\text {ème }}$ siècle. Puis, nous explorons les innovations comptables des dirigeants dans la gestion du résultat. Enfin, nous étudions l'évolution de la législation à une époque où les règles comptables sont d'abord émises par les tribunaux.

\subsubsection{Contradiction entre rétention et prodigalité pour la détermination du résultat}

$\mathrm{Au} 19^{\mathrm{ème}}$, les entreprises industrielles sont constituées à partir de capitaux privés, souvent des capitaux issus de la famille ou des connaissances des dirigeants. Une fois l'investissement initial effectué, l'autofinancement ${ }^{3}$ est privilégié pour assurer la croissance de l'entreprise. Ce double mode de financement (capitaux et autofinancement) laisse apparaître une divergence d'intérêts quant à la détermination du résultat: d'une part, les apporteurs de capitaux souhaitent que leur investissement rapporte et donc que le bénéfice distribuable soit maximal ; d'autre part, les dirigeants, voulant se préserver la marge de manœuvre que leur confère l'autofinancement, préfèrent minimiser leur résultat comptable, tout en préservant leur trésorerie. En effet, les pratiques comptables identifiées dans les activités minières ou métallurgiques au $19^{\text {ème }}$ siècle mettent l'accent sur les flux de trésorerie. Le compte d'exploitation présente le solde des flux, reflet de la santé financière de la firme. Il s'agit d'une comptabilité de caisse, héritière du modèle comptable en recettes et dépenses, appliqué sous l'Ancien Régime (Lemarchand 1995) dans lequel on raisonne en emplois/ressources et non en charges/produits. Si le concept de représentation du patrimoine à travers les états financiers se diffuse, la problématique qui se pose au moment de la décision d'investissement n'est pas de savoir si les produits escomptés permettront de couvrir la dépense - la dette contractée ou le capital investi -, mais si l'on est capable de financer immédiatement cette acquisition à partir des ressources disponibles. Les traitements comptables ne permettent d'ailleurs pas de distinguer la nature de l'opération (investissement ou charge). En l'absence de réglementation comptable, la comptabilité sert donc avant tout aux dirigeants eux-mêmes pour décider s'ils ont ou non la capacité de faire de nouvelles dépenses.

Dès la première moitié du $19^{\text {ème }}$ siècle, il existe une catégorie d'entreprises qui a déjà recours aux capitaux : les Compagnies de chemin de fer. En effet, celles-ci avaient besoin de moyens financiers tels qu'elles ne pouvaient compter sur le réseau personnel des dirigeants ou sur un autofinancement puisque l'exploitation des lignes de chemins de fer n'avait pas encore débuté. Pour attirer des investisseurs, il leur fallait trouver un moyen de rétribuer l'actionnaire

\footnotetext{
${ }^{3}$ L'autofinancement est le premier mode de financement des entreprises au $19^{\text {ème }}$ siècle (Plessis 1996).
} 
privé, qui avait accepté d'investir dans la compagnie avant même l'obtention de tout bénéfice. Cette problématique est explicitée par Lemarchand et Praquin (2005) :

" comment exiger que les commanditaires attendent, avant de rien toucher, jusqu'au moment où l'on pourra savoir si le résultat final sera un bénéfice ou une perte, c'est-à-dire jusqu'au terme d'une société qui peut avoir été formée pour dix, quinze, vingt ans, et même davantage? Quel est le capitaliste qui consentirait à entrer dans une association à de pareilles conditions? D’autre part, si, pendant la durée de la société, des bénéfices ont été distribués, il est à présumer que les commanditaires les ont employés à leurs dépenses journalières ou leur ont donné quelque autre destination; comment exiger dès lors qu'ils en fassent restitution? »(Dalloz, 1859, $\mathrm{v}^{\circ}$ Société, $\mathrm{n}^{\circ} 1385$, repris par Lemarchand et Praquin, (2005, p.19).

A partir de 1890, toutes les sociétés industrielles peuvent avoir recours aux marchés de capitaux, ce qui ouvre des perspectives de croissance très intéressantes (Hautcoeur 2007). Mais ces nouveaux apporteurs de capitaux ne sont plus des personnes physiques issues du réseau social des dirigeants qui acceptent de confier leurs ressources sur la base d'une relation de confiance ${ }^{4}$; il s'agit d'anonymes qui exigent un retour sur investissement. Les dirigeants doivent donc déterminer un résultat comptable présentant des bénéfices permettant de rémunérer les actionnaires par la distribution de dividendes et d'attirer des investisseurs potentiels. Ils souhaitent toutefois continuer à préserver leur autonomie dans la gestion de leur entreprise et donc conserver des possibilités d'autofinancement.

Ainsi, la contradiction entre rétention et prodigalité dans la détermination du résultat, présente dès le $19^{\text {ème }}$ siècle, s'exacerbe à mesure que le financement par les marchés de capitaux s'accroît. La démocratisation de l'actionnariat engendre une pression de plus en plus forte sur la comptabilité.

\subsubsection{Innovations (créativité) comptables pour une gestion du résultat}

Au $19^{\text {ème }}$ siècle, les dirigeants ont une importante marge de manœuvre quant à la reddition des comptes. A cette époque, les amortissements, les réserves et les provisions sont, comme aujourd'hui, les trois postes permettant les meilleurs ajustements.

Ainsi, sous couvert de prudence, certains dirigeants font déjà les choix comptables appropriés pour servir leur intérêt.

La pratique la plus courante concernant les investissements est l'assimilation de ces dépenses à celles d'exploitation. Il s'agit de la pratique de « l'amortissement immédiat » (Lemarchand, 1993) qui permet à la fois de restreindre l'investissement aux moyens disponibles et de pourvoir, par la suite, à leur renouvellement. Parallèlement, avec la baisse du bénéfice liée à cette pratique comptable, la rémunération des actionnaires n'est envisagée qu'une fois les dépenses d'investissement de l'exercice couvertes.

\footnotetext{
${ }^{4}$ Nous verrons par la suite que certains actionnaires issus du réseau de relations, trouvant leur confiance trahie, demanderont réparation devant les tribunaux.
} 
La réserve est également un instrument comptable supplémentaire susceptible de servir les intérêts des dirigeants. L'instruction de 1818 rend obligatoire la constitution d'une réserve visant « à compenser les moins-values potentielles de l'actif immobilisé » (Lemarchand 1993, p.404). Mais contrairement à l'amortissement ou la «retenue pour dépréciation » (Lemarchand 1993, p. 405) qui a pour but de rétablir l'intégrité du capital et est antérieure aux bénéfices, la réserve est un accroissement du capital, prélevée sur les bénéfices. La constitution des réserves légales, statuaires ou autres est laissée à la libre appréciation des gérants, qui disposent d'une importante liberté d'action que seules d'éventuelles revendications, de la part des actionnaires, peuvent venir limiter. Toutefois, cette contrainte est souvent détournée par la constitution de réserves très spécifiques sous des dénominations très diverses, masquant ainsi le véritable caractère de réserve.

Si les différentes réserves librement constituées par les gérants représentent un important moyen de rétention de fonds, d'autres outils comptables peuvent également servir à la constitution de la structure de financement souhaitée, parmi lesquels les provisions. Le terme de provision n'apparaît qu'à la fin du $19^{\text {ème }}$ siècle (Lemarchand 1993). Toutefois, les industriels du $19^{\text {ème }}$ siècle connaissent et maîtrisent déjà parfaitement la dépréciation de l'actif circulant.

$\mathrm{Au}$ travers des traitements comptables mis en œuvre s'affiche toute la créativité dont font preuve les dirigeants pour faire varier dans une grande proportion le rapport entre la «partie visible et cachée du résultat »- pour reprendre l'expression de Lemarchand (1993) - tout au long du $19^{\text {ème }}$ siècle. La constitution de réserves occultes, l'amortissement immédiat ou la dépréciation de l'actif circulant visent l'autarcie financière qui, selon Plessis (1996), devient progressivement un mythe à atteindre pour des entrepreneurs toujours soucieux de trouver des moyens de financement suffisants pour assurer la pérennité de leur firme.

Ainsi, les choix comptables opérés par les industriels ne sont pas des habitudes ou l'héritage de procédés passés mais sont directement liés à la poursuite de leurs intérêts propres. Aussi, avant même l'ouverture des marchés de capitaux, certains actionnaires ne restent pas inactifs devant une minimisation des résultats comptables conduisant à ce qu'ils appellent leur «spoliation».

\subsubsection{Procès, jurisprudences et lois pour la détermination du résultat au $19^{\text {ème }}$ siècle}

En l'absence de réglementation comptable, les tribunaux émettent des jugements sur les affaires qui leur sont soumises, jugements qui font jurisprudences et servent ensuite pour édicter les lois.

Lemarchand et Praquin (2005) font état de plusieurs affaires dans lesquelles les actionnaires, conscients du fait que les dirigeants masquent une partie du résultat au travers des différents mécanismes comptables, intentent des actions en justice pour dénoncer le manque de transparence et la constitution de réserves occultes, les privant ainsi d'une partie de leur rémunération. La confiance qui prévalait lors des apports en capitaux semble avoir été trahie. 
Les juristes du $19^{\text {ème }}$ siècle prônent une évaluation de l'entreprise selon le principe de liquidation, selon lequel il faut en théorie attendre la «mort» de l'entreprise pour procéder à la répartition des bénéfices. Ce principe n'est toutefois pas applicable aux situations présentées par les actionnaires dont les entreprises sont justement en pleine activité. Les actionnaires se verront toutefois déboutés de l'ensemble des procédures judiciaires qu'ils entreprennent, les juges voyant dans les pratiques de minimisation du bénéfice, le résultat d'une gestion prudente de l'entreprise.

La prise de position des juges doit être replacée dans son contexte, afin d'en comprendre la portée. En effet, l'époque est marquée par des scandales financiers et une vague de faillites importantes. Ceux-ci sont essentiellement causés par des entrepreneurs peu scrupuleux qui utilisaient l'absence de réglementation comptable pour gonfler leur résultat et distribuer des dividendes fictifs aux actionnaires. C'est donc pour protéger les créanciers, susceptibles de se laisser abuser par de telles manœuvres frauduleuses (Praquin 2003) que les jugent les déboutent de leurs plaintes. Ils précisent également les modalités de distribution des dividendes : seuls les bénéfices acquis et liquides pourront faire l'objet d'une distribution. La loi de 1856 demande de mettre en œuvre un «inventaire sincère et régulier ». Elle crée le délit de distribution de dividendes fictifs. Toutefois, elle ne précise pas les modalités de détermination du résultat distribuable ni les moyens à mettre en œuvre pour mener cet inventaire Or, ces deux éléments sont justement le socle sur lequel se détermine le «bienfondé » de la distribution des bénéfices.

A partir de la jurisprudence des deux importantes affaires judiciaires qui ont lieu au cours du Second Empire - les affaires Mirès en 1860 et Pereire en 1867-, les contours du concept de résultat distribuable se dessinent progressivement (Lemarchand et Praquin 2005, p. 23) :

"Une distribution de dividendes ne peut donc reposer sur un bénéfice simplement probable mais sur son encaissement. On en revient ainsi partiellement à la conception juridique traditionnelle du résultat de liquidation. La différence essentielle, signe d'une évolution de la réflexion comptable des juristes, tient à ce que la fin de la société n'est plus exigée, mais seulement le dénouement complet - c'est-à-dire l'encaissement-des opérations constitutives du résultat distribuable».

Toutefois, les modalités de détermination du résultat à partir de la différence entre les encaissements et les décaissements de la période sont difficilement applicables du fait de l'essor massif du crédit commercial.

Face à l'inadéquation de cette jurisprudence et la réalité de la vie économique et des affaires, les décisions des tribunaux vont s'inscrire dans une nouvelle voie, entre la prise en compte des résultats anticipés et ceux exclusivement liquides. Si l'exigence de liquidité dans la détermination du résultat est inadaptée en raison de la réalité du cycle d'exploitation et entraîne un retour à la comptabilité de caisse, la prise en compte des plus-values latentes fausse également la réalité du résultat et y introduit une notion d'anticipation et, par définition, d'incertitude. 
La dernière décennie du $19^{\text {ème }}$ siècle est marquée par une ouverture des possibilités de financement externe. Si toutes les entreprises ne peuvent accéder à ces nouvelles opportunités de financement du fait de leur taille ou de leur jeune activité, certaines vont largement les utiliser, tout en essayant de concilier la sauvegarde de leur indépendance et les moyens nécessaires pour procéder aux investissements, leur permettant ainsi de rester compétitifs. Aussi, si jusqu'alors la comptabilité a été mise au service de l'autofinancement, il semble pertinent de s'interroger sur la continuité de telles pratiques en raison de l'enrichissement des modalités d'accès aux capitaux.

Les dirigeants doivent arbitrer entre :

- présenter un résultat favorable en prenant en compte éventuellement les plus-values latentes pour attirer ou fidéliser les actionnaires ;

- minimiser le résultat par la constitution de réserves, parfois occultes, pour consolider la structure financière et éviter l'intrusion de tiers dans la gestion de l'entreprise.

\section{UNE ILLUSTRATION DE LA GESTION DU RESULTAT : LE CAS L'AIR LIQUIDE}

\subsection{Méthodologie}

Nous avons adopté une approche historique pour cette étude de la gestion du résultat. Au-delà de l'introduction de la variable temporelle à l'analyse, l'appel à l'histoire permet de mobiliser diverses sources de données et, en combinant différents niveaux d'analyse, d'appréhender la diversité et la complexité des facteurs intervenant dans la naissance et dans l'évolution des pratiques étudiées. cette méthode est d'ailleurs très utilisée dans les recherches en comptabilité et a démontré la robustesse de ses résultats et la pertinence des analyses qui en découlent.

Pour cette recherche des pratiques de gestion du résultat selon la méthode historique, nous souhaitions étudier, depuis sa création, une entreprise qui avait connu une évolution de ses modes de financement et dont les dirigeants avaient fait des choix comptables sur les postes emblématiques de la gestion des résultats (amortissements, provisions, réserves).

La société retenue est Air Liquide car elle satisfait aux critères évoqués ci-dessus. D'abord, il s'agit d'une société, crée en 1902, issue de la seconde industrialisation et qui fait partie des nouveaux secteurs d'activité qui émergent, au début du $20^{\text {ème }}$ siècle. Ensuite, cette société a connu des évolutions importantes en termes de financement. Enfin, cette société est très innovante pour l'époque et ses actifs, principalement incorporels (brevets), impliquent que les dirigeants ont pu utiliser les accruals pour gérer leur résultat.

Le cas Air Liquide a été étudié de 1902 (date de sa création en société anonyme) à 1939. A cette période, aucune règle ou norme comptables ne viennent contraindre les pratiques comptables des entrepreneurs. Ils ont encore une grande marge de manœuvre dans leurs choix. Ce n'est qu'à partir de la Seconde Guerre mondiale que se dessinent les débuts d'une normalisation comptable en France, le premier plan comptable entrant en vigueur en 1942. 
Les sources mobilisées pour notre étude sont constituées des :

- rapports annuels de la société Air Liquide de 1902 à 1939

- comptes rendus de ses assemblées générales de 1902 à 1945

- registres de présence aux assemblées générales de 1902 à 1939

- listes des souscripteurs établies par la société au moment des augmentations de capital (1902-1913) ou des émissions obligataires (1918-1939)

A des fins de triangulation, nous nous sommes appuyés sur une « enquête bibliographique » qui emprunte à plusieurs disciplines comme l'histoire, les sciences économiques, le droit et la gestion. Ce travail a permis de vérifier la résonance de notre problématique dans la littérature, d'une part et au cours de la période d'étude choisie pour y répondre, d'autre part.

Pour la production de cette description contextualisée de la gestion du résultat nous adoptons une périodisation qui reflète l'objectif assigné secrètement aux modalités de la gestion du résultat. Si sur la première période (1902-1913), la comptabilité vient en appui de la réussite d'un projet entrepreneurial, sur la seconde période (1913-1939), elle sert des intérêts économiques.

\subsection{Modes de financement et comptabilisation des immatériels pour une gestion du résultat}

2.2.1. La phase d'exploration (1902-1905): activer les dépenses immatérielles, une stratégie innovante pour éviter la faillite et obtenir le soutien financier des amis ingénieurs

Georges Claude, diplômé de la cinquième promotion de l'Ecole supérieure de Physique et Chimie industrielle de Paris, découvre en 1896, un procédé permettant le transport et le stockage en toute sécurité de l'acétylène, gaz utilisé pour l'éclairage mais dont les risques d'explosion freinaient jusqu'alors l'utilisation. Ce procédé constitue le premier brevet déposé par Georges Claude dont la liste s'étoffera considérablement au fil des ans.

Ce champ de recherche oriente cet ingénieur vers un thème de recherche très disputé en Europe : le procédé de liquéfaction et distillation de l'air afin d'obtenir de l'oxygène en grande quantité. G. Claude est persuadé qu'il peut améliorer le procédé de Linde et fabriquer de l'oxygène à un coût raisonnable pour un usage industriel. Pour ce faire, il consacre ses nuits à mener des essais dans un entrepôt de la Compagnie française Thomson-Houston qui l'emploie le jour comme ingénieur chargé des recherches techniques sur les retours de courants des tramways. Mais la réalisation de ce projet dépasse ses moyens financiers. Il sollicite alors l'aide financière de Paul Delorme, diplômé de la même école et employé dans la même entreprise au sein de laquelle il occupe un poste de chef du service commercial. Convaincu par le projet de G. Claude, P. Delorme accepte d'apporter son soutien financier, rejoint bientôt par Frédéric Gallier, ancien officier devenu commercial à la ThomsonHouston. Ensemble, ils réunissent, en 1901, une dizaine d'amis et de relations qui acceptent de souscrire aux actions de cette nouvelle société en participations dont le capital s'élève à 50000 francs.

Après de nombreux essais, G. Claude met au point le procédé de liquéfaction de l'air sous pression. Les brevets sont déposés en 1902, date de la création de la société que G. Claude a 
voulu nommer : «L'Air Liquide, société anonyme pour l'étude et l'exploitation des procédés Georges Claude », ayant pour objet :

" - l'étude, l'exploitation, la vente des brevets et inventions de Messieurs Georges et Eugène Claude, relatifs à la liquéfaction industrielle du froid, de l'air liquide et de l'oxygène, à leurs applications ou utilisations ;

- l'étude, l'acquisition, l'exploitation ou la vente de tous brevets, inventions ou procédés ayant trait aux mêmes objets ;

- la production et la vente des produits se rattachant directement ou indirectement à l'objet social. »(Rapport annuel 1902)

Cette société est créée à partir d'un capital de 100000 francs divisé en 1000 actions de 100 francs. Le conseil d'administration est composé des trois co-fondateurs: G. Claude, P. Delorme et F. Gallier et des directeurs de deux entreprises qui fournissent du matériel à $\mathrm{G}$. Claude : Frédéric Brocq, directeur de la Cie Continentale des compteurs et André HenryLepaute, directeur d'une société de fabrication de colonnes de distillation. Parmi les actionnaires à ses débuts, figurent de nombreux ingénieurs comme Charles Burton, d'anciens diplômés de l'Ecole de Physique chimie, des membres de l'Institut comme d'Arsonval, un général en retraite ou encore des grands propriétaires. La présence massive d'ingénieurs dans le financement de cette entreprise s'explique par l'incertitude technologique de l'activité dans la mesure où ils sont les seuls à posséder l'expertise pour en évaluer les chances de réussite et les risques. Les ingénieurs représentent plus de $60 \%$ des actionnaires et se partagent $88 \%$ du capital, soit 340 actions sur les 400 émises à la constitution. Les 12\% restant sont dans les mains de différents propriétaires.

Si le procédé est au point, il ne permet pas encore la fabrication à échelle industrielle de l'oxygène. Mais le temps presse, la concurrence, notamment celle de l'industrie chimique allemande, est vive et les moyens financiers modestes. En effet, outre les apports des actionnaires, les seules ressources financières proviennent d'un music-hall, où l'air liquide est utilisé pour la création de nuages artificiels dans des spectacles.

En 1903, la présentation des travaux de G. Claude à l'Académie des Sciences, permise par d'Arsonval qui est membre de cette prestigieuse institution confirme le sérieux des recherches et apporte une crédibilité supplémentaire permettant de maintenir la confiance des actionnaires qui renouvellent leur fidélité en participant aux différentes augmentations de capital.

En effet, de 1902 à 1905, les augmentations de capital sont annuelles. Le capital passe de 100000 à 500000 francs sur cette période. Ce mode de financement est le seul possible car les établissements bancaires détournent leurs concours de ce type d'investissement, estimant la prise de risque trop élevée. G. Claude et P. Delorme ne peuvent compter que sur la fidélité des actionnaires de la première heure. Toutefois, la fréquence des opérations et l'incertitude sur l'aboutissement du projet rendent difficiles le placement des actions en particulier l'augmentation de capital de 1904 (Jemain 2002). Cette dernière aboutit grâce à la participation d'un industriel Lefebvre-Dibon, qui engage sa fortune personnelle et d'une autre personne M. Babin, dont les caractéristiques sont mal connues. Les souscriptions de ces deux personnes permettent d'éviter la faillite. Le même scénario se reproduit en 1905 où seule l'importante participation de Lefebvre-Dibon permet à nouveau d'éviter la banqueroute. 
L'entrée dans le capital de ce propriétaire modifie légèrement le profil de l'actionnariat de L'Air Liquide. En effet, si les ingénieurs demeurent majoritaires, le pourcentage des propriétaires-investisseurs, c'est-à-dire ceux ayant un patrimoine financier mais pas de formation d'ingénieur, augmente.

Les moyens financiers octroyés par les ingénieurs et les propriétaires-investisseurs sont exclusivement consacrés à des dépenses d'investissement, comptabilisées à leur valeur d'acquisition ou de production au bilan. En 1903, lors du premier exercice comptable, le capital fixe est composé de $99 \%$ du total du bilan. L'actif circulant, composé des postes marchandises, caisse et créances clients est quasi-nul. La composition de l'actif immobilisé est très marquante. Le bilan de 1903 fait apparaître les postes : travaux en cours, petit outillage distinct de matériel et outillage. La présence de ces postes, généralement comptabilisés dans le compte de pertes et profits, suggère une politique "généreuse » d'activation des dépenses. Par ailleurs, dès 1903, plusieurs postes d'immobilisations incorporelles figurent au bilan dont font notamment partie les brevets. Ces derniers présentent un caractère stratégique pour L'Air Liquide (cf. infra raison sociale). Par ailleurs, le rythme auquel les brevets sont déposés par G. Claude en son nom ou en collaboration renforce cette importance.

«Au total, entre 1895 et 1909, on compte 35 brevets (sans compter les certificats d'addition), soit en moyenne 2,26 brevets/an. Jusqu'à la fondation de l'entreprise, on compte 16 brevets et 19 à partir de 1903. Dans cette politique d'invention, G. Claude est manifestement le principal acteur, et son nom figure dans 16 brevets » (Emptoz, 2000, pp.685-686)

La plupart de ces brevets concernent la fabrication de l'air liquide. La cession d'un brevet de Georges Claude à L'Air Liquide est justement à l'origine de la création de la société. A partir de sa création, les dépôts de brevets sont majoritairement réalisés sous le nom de L'Air Liquide, auquel est inévitablement lié le nom de G. Claude. En 1903, ce type d'incorporel représente $12 \%$ de l'actif immobilisé.

Les brevets ne sont pas les seuls éléments incorporels, les frais de constitution et d'augmentation de capital sont également immobilisés et prennent une importance considérable compte tenu de la fréquence de ces opérations. Figurent également au bilan les frais d'essais dès 1903 .

Les bilans de la société L'Air Liquide étonnent quant à leur composition. La présence d'actifs incorporels, dont la dépréciation n'est pas énoncée, est innovante au début du $20^{\mathrm{ème}}$ siècle, période durant laquelle ces dépenses représentent des non valeurs, habituellement comptabilisées dans le compte de pertes et profits. Ce mode de comptabilisation permet de ne pas grever lourdement le résultat et ainsi minimiser les pertes réalisées. En effet, le passage immédiat au compte de pertes et profits de ces dépenses entraînerait de lourds déficits, aux conséquences fortes sur les premières années d'activité dans le cas d'un report éventuel sur les exercices suivants. La volonté de minimiser les pertes est également observable par l'absence de constat de dépréciation sur cette période. Cette absence n'est pas le fait d'une méconnaissance des pratiques comptables mais répond à l'objectif de minimisation des pertes. L'absence d'amortissement enlève ainsi une charge supplémentaire dans le solde du compte de pertes et profits.

2.2.2. La phase d'exploitation (1906-1913) ou gonfler artificiellement le résultat en vue de séduire le plus d'actionnaires à la Coulisse 
En mai 1905, G. Claude atteint son objectif et met au point l'appareil industriel capable de produire le gaz liquide en grande quantité. A partir de cette date, la phase d'exploitation peut commencer. Les premières usines sont construites en France. P. Delorme privilégie une stratégie de proximité en raison du poids des bouteilles d'oxygène et du coût du transport induit. Les installations de l'Air Liquide se multiplient donc près des centres de consommation d'oxygène.

Les dirigeants de L'Air Liquide déposent des brevets partout dans le monde afin de protéger les droits industriels de la société à l'étranger. La concurrence est vive sur ce type d'inventions, comme le prouve un conflit qui oppose L'Air Liquide et son concurrent le physicien allemand, Linde, au sujet d'un brevet dont les deux parties s'accordent la primeur. La stratégie de proximité est également déployée à l'international: construction d'installations et exploitation des procédés en Belgique en 1906, au Japon en 1907, en Grande-Bretagne, en Italie, au Canada ou encore dans plusieurs pays asiatiques au cours des années suivantes. Pour s'étendre à l'échelle mondiale, la stratégie de l'Air Liquide se concentre sur la production et la vente d'oxygène via ses propres installations ou en échange de redevances versées sur l'exploitation de brevets.

L'exploitation du procédé de G. Claude découvert en 1905 ne met pas un frein à la politique d'innovation. Celle-ci obéit à une gestion prudente. La phase d'exploration de nouveaux procédés ou inventions, souvent annexes au métier de base de L'Air Liquide, est réalisée par des sociétés distinctes dans lesquelles cette dernière détient une faible participation. Dès que l'invention ou le procédé est mis au point, c'est-à-dire au moment du passage à la phase d'exploitation, le besoin de financement est tel que L'Air Liquide augmente sa participation, allant jusqu'à incorporer la société en question. Le financement de ces nouveaux investissements se réalise toujours par des augmentations de capital selon un rythme annuel. Toutefois, celles-ci s'opèrent depuis 1906 sans difficulté, l'action L'Air Liquide n'étant plus considérée comme un placement «à risque ». Ceci est confirmé par l'entrée dans le capital de la Banque Vernes, établissement de la Haute Banque, qui apporte tout son prestige et sa renommée à cette jeune entreprise, même si sa participation dans le capital est relativement faible.

L'exercice de 1905 a fait apparaître le premier bénéfice et dès l'exercice suivant, il est proposé une distribution de dividendes à hauteur de $4 \%$ du capital versé après dotation à la réserve légale de $5 \%$ du résultat net (Rapport annuel 1906). L'affectation du résultat est notifiée pour la première fois dans les statuts de la société en 1909, après sa cotation à la Coulisse en 1908. Auparavant, l'affectation du résultat sert d'autres intérêts. En effet, l'affectation des premiers bénéfices en 1905 fait apparaître un report à nouveau créditeur d'un montant de près de 4000 francs courants. Or ce montant ne figure pas dans le report à nouveau de l'exercice suivant et n'est pas intégré au bénéfice distribuable. Les dirigeants ont comptabilisé cette somme dans le compte de résultat de l'exercice 1906, venant donc alimenter le résultat brut de l'exercice. Ainsi, le résultat de 1906, tel qu'il est présenté, compte en fait deux années de bénéfices (1905 et 1906). Ce type de mesure reflète le souci des dirigeants de montrer une image des plus flatteuses des débuts de la société. A partir de la cotation à la Coulisse, l'affectation du résultat se formalise. La dispersion de l'actionnariat de L'Air Liquide est une conséquence de cette introduction sur le second marché. Petit-Konczyk (2003) précise dans son étude sur l'actionnariat de L'Air Liquide que le nombre d'actionnaires est multiplié par 16 entre 1908 et 1912. Conjointement, la souscription 
moyenne diminue et est divisée par 13 sur cette même période. Avant même son entrée sur le marché boursier officiel en 1913, cette société se caractérise par les prémices d'une dispersion progressive de son actionnariat. Les registres de présence aux assemblées générales nous donnent quelques indications sur la composition de l'actionnariat. Si la vingtaine de personnes ayant participé à la création de L'Air Liquide sont toujours présentes, elles sont à présent entourées d'un nombre croissant de petits porteurs. Par ailleurs, la Banque Chauve rejoint la Banque Vernes dans le capital de celle-ci. Si, à chaque augmentation de capital, les actions L'Air Liquide trouvent preneurs sans difficulté, c'est notamment en raison de la politique généreuse de rémunération des actionnaires; au lieu de se constituer des réserves, les dirigeants choisissent de distribuer le résultat en constante augmentation au cours de cette période.

Les choix comptables opérés appuient cette politique de rémunération des actionnaires. Tout d'abord, la composition des bilans met en évidence l'importance de l'actif immobilisé. L'ensemble des dépenses d'investissement sont comptabilisés à l'actif y compris les dépenses immatérielles. L'actif circulant est quasi-inexistant en l'absence d'une réelle activité. Ce poste se renforce au fil des années mais demeure toujours relativement faible comparativement à l'actif immobilisé. Il représente respectivement 33, 81\% et 53,36\% en 1913. L'importance de l'actif fixe reflète l'effort d'investissement mené par les dirigeants autant sur le marché domestique qu'à l'international. Mais cette importance traduit également une politique d'activation des dépenses. En effet, on retrouve sur cette période des postes tels que les études de laboratoire de G. Claude ou encore le Petit outillage distinct du poste Matériel et outillage. La présence de ces postes laisse suggérer le maintien d'une politique généreuse d'activation des dépenses. En effet, ces postes sont difficilement assimilables à des dépenses d'investissement et sont généralement comptabilisés à la charge de l'exploitation dans le compte de pertes et profits. Un tel positionnement de la part des dirigeants semble guidé par la volonté de ne pas grever le résultat comptable sur une période trop empreinte d'incertitude.

Un constat similaire peut être dressé pour les dépenses immatérielles Dès sa création, L'Air Liquide innove en comptabilisant les dépenses immatérielles dans l'actif fixe. En effet, ces dépenses sont, à l'époque, considérées comme des actifs fictifs aux retombées incertaines. Les inclure dans l'actif conduit à gonfler artificiellement le résultat distribuable. Or, la distribution de dividendes fictifs constitue un délit depuis la loi de 1856. Dès lors, en activant ces dépenses, les dirigeants de 1'Air Liquide prennent un risque et sont en contradiction avec les pratiques des autres entrepreneurs. A côté des brevets présents à l'actif dès 1903, figurent les frais de constitution et d'augmentation de capital qui prennent une importance croissante en raison de la fréquence des opérations en capital. Leur activation n'est pas un obstacle à la distribution de dividendes. Les immobilisations incorporelles comportent également les frais d'essais, sans aucune explication sur le contenu de ce poste.

En 1908, les actifs incorporels représentent 96,67\% de l'actif fixe. Leur présence et leur importance laissent suggérer la mise en œuvre d'une politique de gestion du résultat durant cette période de forte incertitude où il est nécessaire de rassurer les investisseurs. Ceci se confirme avec l'étude de la seconde période où l'on observe une forte rupture dans la gestion des investissements incorporels. 
A la veille du premier conflit mondial, L'Air Liquide est introduite sur le marché financier officiel. Pendant la guerre, il y aura une seule augmentation de capital, en 1917. L'émission de 40000 actions de 200 francs chacune, comprenant une prime d'émission égale à la valeur nominale se fait sans difficulté mais érode davantage le contrôle des fondateurs dans la gestion de cette société. Les dirigeants émettent pour la première fois, en 1918, des obligations pour un montant de 7500000 francs. La cotation en Bourse a donné la visibilité et l'assurance pour le placement de ces titres de dette. Les dirigeants de L'Air Liquide en profitent pour recourir annuellement à ce mode de financement entre 1918 et 1920.

Durant la première guerre mondiale, L'Air Liquide participe à l'effort de guerre en alimentant en oxygène et acétylène le secteur métallurgique. Les besoins issus de la guerre génèrent pour cette société un accroissement de ses capacités de production d'une part, et la création de nouveaux centres de fabrication d'autre part. A la fin des hostilités, l'implantation des usines en France et à l'étranger est renforcée. Durant le conflit, Georges Claude ne cesse de proposer de nouvelles idées pour aider les armées. En 1919, pour doter l'ingénieur de moyens supplémentaires pour ces travaux sur la synthèse de l'ammoniac, une nouvelle société est créée, la Société Chimique de la Grande Paroisse. Son capital est réparti entre L'Air Liqude et la Manufacture Saint-Gobain, Chauny et Cirey. Cette société a pour objet de «doter la France d'une industrie des engrais - et, incidemment, de fabrication d'explosifs » (Jemain, 2002, p.50). Mais l'ensemble des propositions de G. Claude ne trouve pas l'attention espérée, faisant naître auprès de leur créateur un sentiment d'amertume et de rancœur qui se révèlera lors du second conflit mondial.

Durant cette période, les choix comptables demeurent quasi identiques à ceux de la période précédente. Les actifs fixes occupent toujours une place prépondérante dans l'actif total. L'actif circulant oscille en moyenne autour de $35 \%$ du total du bilan. A la veille de la Grande Guerre, l'activité de L'Air Liquide commence à se consolider et les investissements corporels acquièrent de ce fait une place prépondérante dans l'actif fixe. En revanche, le poids des dépenses incorporelles dans l'actif fixe connait quant à lui un fléchissement en raison d'un changement comptable. Les nouveaux brevets ou encore les frais d'essais ne sont plus portés à l'actif mais sont comptabilisés en charges. Seul les frais d'augmentation de capital demeurent mais sont à présent amortis sur la prime d'émission.

Durant cette période, comme dans la précédente, les actifs fixes occupent toujours une place prépondérante dans l'actif total. L'actif circulant oscille en moyenne autour de $35 \%$ du total du bilan. Toutefois, la constitution de l'actif fixe est modifiée. En effet, à la veille de la Grande Guerre, l'activité de L'Air Liquide commence à se consolider et les investissements corporels acquièrent de ce fait une place prépondérante dans l'actif fixe. En revanche, le poids des dépenses incorporelles dans l'actif fixe connait quant à lui un fléchissement en raison d'un changement comptable notoire. En effet, les nouveaux brevets ou encore les frais d'essais ne sont plus portés à l'actif mais sont comptabilisés en charges. Seuls les frais d'augmentation de capital demeurent à l'actif, mais ils sont à présent amortis sur la prime d'émission.

Malgré le passage en charges de dépenses auparavant activées, la distribution de dividendes ne connaît pas de ralentissement. Jusqu'à la sortie de la Première Guerre mondiale, le 
bénéfice net est quasi-exclusivement distribué aux actionnaires sous forme de dividende. Outre les dispositions légales, les dirigeants ont rarement recours aux différents instruments permettant une rétention plus forte des profits. Les courbes représentatives de l'évolution des bénéfices bruts et des dividendes distribués sont quasi-confondues jusqu'en 1918. La rémunération des actionnaires s'inscrit donc toujours comme une priorité. Cette pratique est peu courante pour les jeunes sociétés de l'époque. En effet, généralement, les actionnaires des sociétés anonymes ou en commandite laissent à la disposition des dirigeants le montant des dividendes déterminés. Ces sommes sont placées sur des comptes courants rémunérés dont le dirigeant peut disposer en l'absence d'autres ressources financières. Les dirigeants n'ont pas recours à de telles pratiques. Les actionnaires ayant participé financièrement aux successives opérations en capital dans un contexte de forte incertitude conçoivent les premiers dividendes comme la concrétisation de la réussite du projet scientifique. Pour les dirigeants, ce dividende constitue une reconnaissance de la fidélité et de l'investissement des actionnaires durant la phase d'exploration.

2.2.4. Retour aux fondamentaux (gaz industriels) et développement international (19201939): recherche d'un équilibre entre constitution d'autofinancement et rétribution de l'actionnaire afin d'éviter les conflits d'agence

Le premier conflit mondial a été pour L'Air Liquide un détonateur d'activité. A partir de 1920, les augmentations en capital reprennent selon un rythme plus soutenu dans la mesure où les ressources financières sont nécessaires pour accroître le développement de la société à l'international. Après avoir intensifiée sa présence sur le marché domestique et le continent européen (Belgique, Italie, Espagne), la société L'Air Liquide s'implante notamment dans différents pays méditerranéens (pays du Maghreb, Egypte). Par ailleurs, le mouvement d'absorption de filiales s'intensifie au cours de cette décennie conformément à la stratégie de croissance externe souhaitée par Paul Delorme. La création de sociétés, comme par exemple la Société Chimique de la Grande Paroisse, créée en 1919 et chargée de commercialiser les travaux de G. Claude sur la synthèse de l'ammoniac, obéit à des règles prudentes, à savoir une certaine vigilance quant à l'exploitation des nouvelles découvertes de cet ingénieur. Le dirigeant, Paul Delorme, isole ces projets dans des sociétés distinctes et augmente progressivement la participation de L'Air Liquide pour les intégrer finalement intégralement en fonction des succès réalisés. C'est ainsi qu'en 1923, sont intégrées la Société centrale des industries de l'air liquide et de l'azote, la Société acétylène dissous et applications de l'acétylène, la société d'oxygène et d'acétylène du Japon, la société nord-africaine de gaz comprimés et enfin la Société égyptienne de gaz comprimés. Au début de cette nouvelle décennie, Paul Delorme souhaite se focaliser sur le métier de base, c'est-à-dire les gaz industriels. L'Air Liquide se recentre donc sur ses fondamentaux laissant de côté l'ensemble des projets développés par G. Claude durant la Guerre comme par exemple les travaux effectués sur la synthèse d'ammoniac.

L'augmentation de capital réalisée en 1928 se distingue de celles réalisées jusqu'alors car pour la première fois, il ne s'agit pas d'obtenir de nouvelles ressources financières mais de se protéger contre d'éventuelles prises de contrôle de groupes étrangers. Dans cet objectif, le transfert de réserves en capital a entraîné la création d'actions à vote privilégié. Les nombreuses augmentations de capital ont inévitablement entraîné une baisse de leur participation dans le capital de la société, érodant ainsi le contrôle des dirigeants-fondateurs. 
La création d'actions à vote plural est un moyen d'une reprise de contrôle de ces derniers. A partir de cette date, les augmentations de capital se raréfient : celle de 1933 vise à supprimer les actions à vote plural conformément à la loi du 13 novembre 1933 et celle de 1937 est programmée pour le financement du transfert du siège social de la rue Saint-Lazare au Quai d'Orsay. Le ralentissement des augmentations de capital peut également s'expliquer par la volatilité accrue des marchés financiers dans un contexte de crise économique. Les dirigeants ne se détournent pas complétement du marché, ils utilisent d'autre supports comme l'émission de titres de dette pour obtenir des ressources financières supplémentaires. Les dirigeants y trouvent refuge en 1929 et 1930 pour un montant respectif de 75000000 francs. La dernière aura lieu en 1939 mais est d'une moindre ampleur.

En termes de financement, la période étudiée est marquée par la montée en puissance de l'autofinancement. Relativement modeste jusqu'alors, les dirigeants commencent à mettre en œuvre les moyens nécessaires à la constitution de ce mode de financement, qui surpassera les apports des actionnaires seulement à partir de 1934. Jusqu'alors, les dirigeants n'ont pas forcé la constitution de cette ressource par le recours à des instruments comptables permettant la rétention de bénéfices. Au début des années 1920, ce mode de financement se forme en raison de la forte croissance de l'activité mais va ensuite être renforcé à partir de 1925 par certains choix comptables.

Le décollage industriel de la société à la sortie de la guerre, visible par la forte croissance du chiffre d'affaires entraîne une évolution dans la politique comptable appliquée par les dirigeants. Cette évolution est notamment observable à travers les changements opérés dans le traitement comptable des dépenses d'investissement. En 1925, plus aucune immobilisation incorporelle ne figure au bilan de L'Air Liquide. Ces actifs sont désormais maintenus pour mémoire dans le patrimoine de la société. Les dépenses d'investissement immatériel n'ont cependant pas cessé ; les innovations mises au point par les différents ingénieurs dont G. Claude figurent toujours au cœur de l'activité de cette société et sont immédiatement brevetés en raison d'une concurrence internationale intense. Si les dépenses immatérielles sont toujours présentes, leur mode de comptabilisation a évolué. Le bénéfice brut peut désormais supporter l'ensemble de ces charges. Ainsi les dépenses relatives aux nouveaux brevets déposés ou les frais d'essai ne sont plus comptabilisés à l'actif mais sont immédiatement enregistrés dans le compte de pertes et profits.

Par ailleurs, jusqu'à la sortie de la Première Guerre mondiale, le bénéfice net après amortissement est quasi-exclusivement distribué aux actionnaires. Sans remettre en cause le montant des dividendes distribués annuellement, le décollage industriel dans les années 1920 de L'Air Liquide permet aux dirigeants de mener de concert une politique généreuse à l'encontre des actionnaires et les prémices d'une politique d'autofinancement. En 1923, un poste intitulé «Fonds d'amortissement» est créé en remplacement de celui qualifié de «Réserve pour amortissement». Les dotations à ce poste constituent une rétention de bénéfices, qui peuvent être analysées comme un amortissement extraordinaire en supplément de l'amortissement économique comptabilisé annuellement. La même année, ce poste est doté du reliquat des réserves d'amortissement des sociétés absorbées. En 1925, les membres du conseil d'administration décident de doter exceptionnellement ce poste par prélèvement sur le bénéfice afin de le «mettre en rapport avec l'importance accrue des immobilisations et du capital » (rapport annuel, 1925). Cette opération est réitérée en 1926 lors de l'affectation du résultat. Sur la même année, il est prévu d'affecter une partie du résultat net au poste 
«Réserve spéciale ». Cette affectation n'est pas justifiée dans le rapport annuel. En 1922, l'autofinancement retrouve son niveau d'avant-guerre, soit environ $27 \%$ du total du passif. Cette tendance haussière se confirme jusqu'à la fin des années 1920. En 1927, cette ressource financière représente plus de $30 \%$ du total du bilan. Les rétentions sur le résultat sont plus fortes avec d'importantes dotations au poste «Réserve pour amortissement ». Les premiers signes de la crise économique au début des années 1930 semblent perceptibles sur l'activité économique de L'Air Liquide. Une légère baisse de l'autofinancement dans le total du bilan est observable sur les exercices 1929 et 1931. Toutefois, cette courbe s'inverse à partir de 1932, l'autofinancement enregistre sur la fin de la période étudiée une forte croissance. En 1939, il représente 52\% du total du bilan alors qu'il n'était qu'à hauteur de 31\% en 1931 .

A la veille du second conflit mondial, L'Air Liquide dispose d'importantes ressources financières internes. Cependant, cette forte augmentation n'est pas corrélée à une hausse du résultat car celui-ci connaît entre 1931 et 1936 un léger recul. En outre, les prémices d'une politique de désinvestissement semblent observables à travers la diminution des dotations comptabilisées sur cette période. En revanche, la baisse de rémunération versée aux actionnaires sous la forme de dividendes entre 1931 et 1939 permet de compenser la diminution du résultat et d'assurer la croissance de l'autofinancement. La diminution du bénéfice distribué semble essentiellement être à l'origine de cette augmentation dans la mesure où les dirigeants ne recourent pas sur cette période à une rétention des profits à travers la comptabilisation de réserves ou de provisions. C'est la première fois que l'on observe une baisse du montant distribué aux actionnaires au début de cette période. Afin d'éviter toute contestation, les dirigeants mettent l'accent sur le contexte économique de la période au cours de cette période. Toutefois, l'actionnariat de L'Air Liquide apparait comme fidèle et peu versatile. Ceci est confirmé par le portrait dressé par l'ouvrage édité à l'occasion des 100 ans de L'Air Liquide. Cet ouvrage souligne la cote de confiance dont bénéfice l'action de la société à la sortie de la Première Guerre mondiale. La politique de distribution des dividendes mis en place par les dirigeants constitue un élément explicatif des caractéristiques de son actionnariat.

A la veille de la Seconde Guerre mondiale, L'Air Liquide compte 12000 salariés répartis dans 200 usines à travers le monde. Au moment où éclate le conflit mondial, il ne s'agit plus de croître géographiquement mais de conserver les brevets et installations réalisés à l'étranger. La crainte que les usines tombent dans les mains des concurrents est omniprésente, durant tout le conflit ainsi qu'à la Libération. En 1945, c'est une toute autre menace qui pèse sur l'Air Liquide car cette dernière figure parmi les entreprises à nationaliser en raison de la vague de nationalisation décidée par l'Etat. Il faudra l'appui des différentes relations de Jean Delorme, fils de P. Delorme, établies avec des hauts personnages de la Résistance, pour éviter que L'Air Liquide ne tombe sous la coupe de l'Etat. En effet, Jean Delorme, entré dans la société en 1924, dont il est le directeur général en 1935, a démissionné de ses fonctions pendant la guerre pour servir la Résistance. Son engagement contre l'occupant sera un atout majeur pour lutter contre l'assimilation entre L'Air Liquide et Georges Claude, dont les déclarations publiques de soutien à l'envahisseur se sont multipliées jusqu'aux dernières heures de l'Occupation. L'inventeur sera contraint de démissionner de son mandat d'administrateur en 1943. 


\section{DISCUSSION ET CONCLUSION}

De 1902 à 1939, Air Liquide a connu deux périodes majeures au cours desquelles les dirigeants ont utilisé la comptabilité pour servir leur stratégie.

Le tableau 1 reprend les principaux objectifs des dirigeants, les modes de financement et les choix comptables aux différentes phases, à partir desquels les pratiques de gestion du résultat peuvent être analysées. 


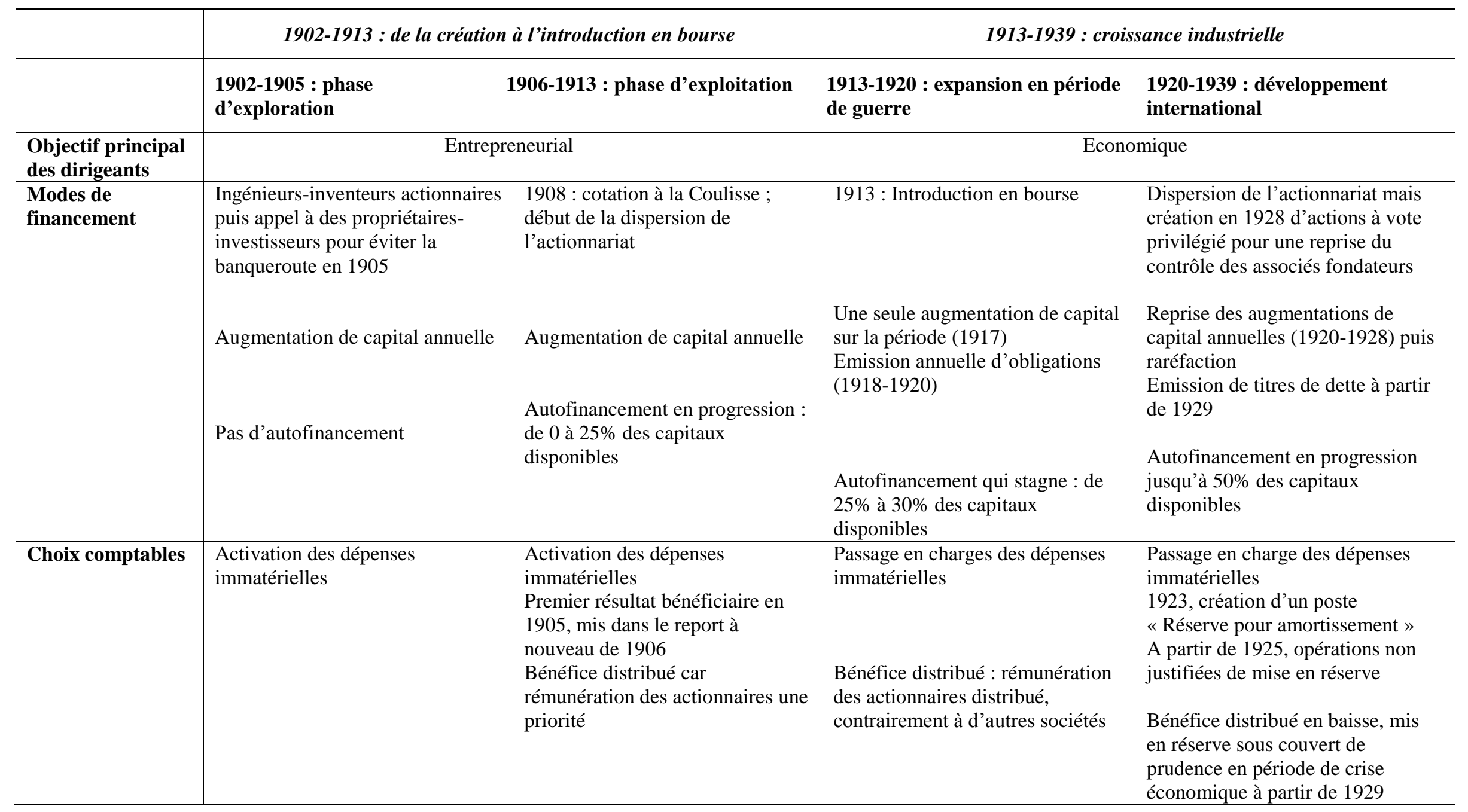

Tableau 1 : Objectifs, modes de financement et choix comptables à L'Air Liquide (1902-1939) 


\section{2-1913 : une gestion du résultat pour favoriser le projet innovant}

Entre 1902 et 1905, l'objectif premier des fondateurs est entrepreneurial. Les capitaux engagés par la jeune équipe d'ingénieurs qui croit au projet se révèlent rapidement insuffisants pour poursuivre les recherches sur la liquéfaction de l'air. Les augmentations de capital annuelles servent à éviter à chaque fois la banqueroute et les nouveaux entrants s'apparentent davantage à des mécènes qu'à des actionnaires souhaitant tirer profit de leur placement. En termes de gestion du résultat, l'activation des dépenses immatérielles est une nécessité pour présenter un résultat pas trop déficitaire, l'objectif étant d'arriver à convaincre des amis de continuer à investir pour poursuivre l'aventure. Cette pratique est tout à fait contraire aux recommandations prudentielles de l'époque.

En 1905, le procédé permet enfin de produire l'air liquide à l'échelle industrielle ; l'activité peut se déployer. L'objectif premier des dirigeants-fondateurs, G. Claude et P. Delorme, est toujours entrepreneurial. Ils mettent en place une stratégie industrielle de proximité, mais protègent l'innovation en déposant leur brevet dans de nombreux pays. La méthode juridique pour ce déploiement est astucieuse et, cette fois, prudente. A chaque nouvelle invention, une société est créée et, une fois le procédé au point, absorbée progressivement par l'Air Liquide. $\mathrm{Au}$ cours de cette période, les besoins de financement pour passer en phase de production sont énormes, sans commune mesure avec les besoins initiaux de recherche. La recherche de nouveaux actionnaires est active. Les comptes doivent présenter le meilleur résultat possible pour donner une image flatteuse de l'entreprise. Dans cette optique, les dépenses immatérielles continuent d'être activées. Le premier résultat bénéficiaire de 1905 est intégré dans le report à nouveau de l'année suivante afin de gonfler artificiellement le résultat de 1906. Les actionnaires initiaux, impliqués dans le projet, sont ainsi prêts à renoncer à leurs dividendes pour poursuivre l'aventure avec d'autres. A partir de 1908, la cotation à la coulisse facilite les augmentations annuelles de capital, même si le capital commence à se disperser : un grand nombre de petits actionnaires viennent compléter les vingt actionnaires initiaux.

La hiérarchie des modes de financement utilisés par L'Air Liquide infirme les idées reçues sur le financement de l'industrie à cette époque. Loin de constituer le mode de financement prédominant, l'autofinancement est largement devancé par les ressources obtenues lors des multiples augmentations de capital. Si ce mode de financement est contraint par l'objet de la société durant les premiers exercices, il s'affirme en phase d'exploitation. Compte tenu de la fréquence des augmentations de capital et des limites de leur fortune personnelle, la baisse du degré de contrôle des fondateurs est inévitable. Pour autant, cela ne constitue pas un frein pour ces derniers dans la recherche de capitaux sur le marché financier.

La configuration actionnariale des débuts de l'Air Liquide est assez proche de ce que Wang (2006) analyse à travers la théorie de l'agence concernant les firmes familiales. Pour autant, les résultats observés sont très différents de ceux de Wang (2006). Pour ce dernier, deux effets contradictoires sont en jeu: d'abord, par l'effet d'enracinement, les dirigeants devraient profiter de l'asymétrie d'information aux dépends des autres actionnaires; par l'effet d'alignement, ils devraient présenter des résultats de meilleure qualité (moins d'accruals) pour ne pas entacher leur réputation. D'après notre étude, la gestion du résultat pratiquée par les fondateurs n'est pas destinée à maximiser leur intérêt économique. Ces actionnaires- 
dirigeants poursuivent une stratégie entrepreneuriale très marquée, quitte perdre une partie du contrôle de la société alors qu'ils ont pris tous les risques au départ. Bien qu'il y ait une relation d'agence entre actionnaires et dirigeants, les catégories de la théorie politicocontractuelle ne sont pas pertinentes sur cette première période. La réussite du projet est à ce moment plus forte que l'enrichissement économique. Si les dirigeants distribuent des dividendes, c'est d'abord pour fidéliser leurs actionnaires et créer un effet de réputation afin que d'autres rejoignent l'aventure. Cette pratique était d'ailleurs risquée puisque certains auraient pu taxer les dirigeants de délits de dividendes fictifs (loi de 1856), les dividendes étant distribués sur la base de l'activation des dépenses incorporelles.

\section{3-1939 : une gestion du résultat pour profiter des retombées financières}

La deuxième période peut davantage s'analyser à travers la théorie de l'agence puisque les actionnaires-dirigeants vont alors tout faire pour maximiser leur intérêt économique.

La période 1913-1920 est singulière puisqu'il s'agit d'une expansion liée à la Première Guerre mondiale. A partir de l'introduction en bourse, une seule augmentation de capital a été observée en 1917, mais on peut penser que ceci est dû aux difficultés à mobiliser de nouveaux actionnaires dans un contexte troublé. En outre, les dirigeants veulent freiner la dispersion du capital et préfèrent émettre des obligations pour financer leur activité. La stratégie des dirigeants change et leurs pratiques comptables également. En effet, à partir de 1913, les dépenses immatérielles seront systématiquement passées en charge et le bilan de la société l'Air Liquide, qui était jusqu'à présent atypique, rejoint le modèle traditionnel des sociétés industrielles: l'objectif des dirigeants est de minimiser le résultat distribuable afin de favoriser l'autofinancement, qui représente alors environ $30 \%$ des capitaux disponibles. Toutefois, on observe que les dirigeants continuent de distribuer des dividendes, contrairement à d'autres sociétés de la même époque pour poursuivre la fidélisation de leur actionnariat.

La période 1920-1939 s'inscrit dans la continuité de la précédente. Les augmentation de capital annuelles reprennent entre 1920 et 1928, et sont stoppées net. En effet, malgré les efforts pour conserver un noyau dur, la dispersion progressive du capital conduit en 1928 presqu'à la perte du contrôle de la société au profit de groupes étrangers. La création d'actions à vote plural permet de garantir le contrôle aux dirigeants-fondateurs. En termes de gestion du résultat, les dirigeants utilisent peu à peu tout ce qu'ils peuvent pour minimiser le résultat comptable : les dépenses immatérielles sont passées en charge ; à partir de 1923, ils créent un poste « réserve pour amortissement »; à partir de 1925, une succession d'opérations de mise en réserve ne seront pas justifiées. Malgré une augmentation constante du chiffre d'affaires, le résultat comptable connaît un certain recul et les dividendes versés aux actionnaires diminuent entre 1931 et 1936, sous couvert de prudence dans un contexte de crise économique. L'autofinancement dépasse alors les 50\% des capitaux disponibles en 1939. Malgré cette pratique, l'actionnariat demeure fidèle.

En diminuant le recours aux marchés au bénéfice du financement interne, les dirigeants font des choix comptables susceptibles d'attiser les plaintes des actionnaires à qui l'ont soustrait une partie de leur rémunération. Pour autant, aucune plainte d'actionnaires n'est enregistrée sur cette période. Les dirigeants s'assurent d'un niveau relativement élevé de dividendes pour éviter tous conflits d'agence. Par ailleurs, favoriser le financement interne leur permet ne pas 
accentuer l'éclatement de leur actionnariat déjà considérablement diffus. Au travers de ces pratiques, les dirigeants recherchent leur indépendance et la maximisation de leur intérêt économique.

En conclusion, plus les actionnaires sont loin du projet, plus ils cherchent à être rémunérés. Ceux qui sont proches du projet ont d'autres motivations. 


\section{BIBLIOGRAPHIE}

Ali, A., Chen, T-Y., Radhakrishnan, S. (2007). Corporate disclosures by family firms. Journal of Accounting and Economics 44(1-2): 238-286.

Beattie, V., Brown, S., Ewers, D., John, B., Manson, S. Thomas, D., Turner, M. (1994). Extraordinary items and income smoothing: A positive accounting approach. Journal of Business Finance and Accounting 21(6): 791-811.

Buckmaster, D. (1992). Income smmthing in accounting and business literature prior to 1954. The Accounting Historians Journal 19(2): 147-173.

Chalayer, S. (1994). Le lissage des résultats. Elements explicatifs avancés dans la littérature. Comptabilité Contrôle Audit 12(1) : 37-65.

Degeorge, F., Patel, J., Zeckhauser, R. (1999). Earnings management to exceed tresholds. Journal of Business 72(1): 1-35.

De La Bruslerie, H. (2014). Le contrôle dans les entreprises est-il compatible avec la responsabilité limitée des actionnaires contrôlants? In L'état des entreprises 2014 (Ed, Dauphine Recherches en Management). Paris : La Découverte : 56-68.

Emptoz, G. (2000). La création de L'Air Liquide au début du XXe siècle. In Créateurs et créations d'entreprises de la révolution industrielle à nos jours (Ed. Marseille J. Paris: ADH : 677-692.

Faccio, M., Lang, L. (2002). The ultimate ownership of western European corporations. Journal of Financial Economics 65(3): 365-395.

Hautcoeur, P.C. (2007). Le marché financier français au XIXème siècle. Paris : Publications de la Sorbonne. Vol. 1.

Holthausen, R.W., Larcker, D., Sloan, R. (1995). Annual bonuse schemes and the manipulation of earnings. Journal of Accounting and Economics 19(1): 29-74.

Jeanjean, T. (2001). Incitations et contraintes à la gestion du résultat. Comptabilité Contrôle Audit 7(1) : 62-76.

Jemain, A. (2002). Les conquérants de l'invisible: Air Liquide, 100 ans d'histoire. Paris : Editions Fayard.

Lemarchand, Y. (1993). Du dépérissement à l'amortissement : enquête sur l'histoire d'un concept et de sa traduction comptable. Nantes : Ouest Editions.

Lemarchand, Y. (1995). Style mercantile ou mode des finances, le choix d'un modèle comptable dans la France d'Ancien Régime. Annales HSS 1 : 159-182.

Lemarchand, Y., Praquin, N. (2005). Falsifications et manipulations comptables - La mesure du profit, un enjeu social (1856-1914). Comptabilité Contrôle Audit 11(Juilllet): 15-34.

Mard, Y., Marsat, S. (2012). Gestion des résultats et structure de l'actionnariat: le cas français. Comptabilité Contrôle Audit 18(3) : 11-42.

Marmousez, S. (2009). Gestion du résultat. In Encyclopédie de Comptabilité, Contrôle de Gestion et Audit (Ed, Colasse, B.). Paris : Economica : 851-860.

Moses, O.D. (1987). Income smoothing and incentives: Empirical tests using accounting changes. The Accounting Review 62(2): 358-377.

Petit-Konczyk, M. (2003). Le financement d'une start-up : L'Air Liquide 1898-1913. Finance Contrôle Stratégie, 6(4) : 25-58.

Plessis, A. (1996). Le financement des entreprises. In Histoire de la France industrielle (Ed, Lévy-Leboyer, M.). Paris : Editions Larousse, 126-159.

Praquin, N. (2003). Comptabilité et protection des créanciers (1807-1942) : une analyse de la fonction technico-sociale de la comptabilité. Doctorat en sciences de gestion, Paris: Université Paris-Dauphine. 
Smith, E. (1976). The effect of separation of ownership from control on accounting policy decisions. The Accounting Review 51(4): 707-723.

Stolowy, H., Breton, G. (2003). La gestion des données comptables : une revue de la littérature. Comptabilité Contrôle Audit 9(1) : 195-213.

Vidal, O. (2010). Gestion du résultat pour éviter de publier une perte : les montants sont-ils marginaux ? Comptabilité Contrôle Audit 16(3) : 11-39.

Wang, D. (2006). Founding familiy ownership and earnings quality. Journal of Accounting Research 44(3): 619-655. 